\title{
TeV Neutrinos from Successful and Choked Gamma-Ray Bursts
}

\author{
Peter Mészáros ${ }^{1}$ and Eli Waxman ${ }^{2}$ \\ ${ }^{1}$ Pennsylvania State University \\ ${ }^{2}$ Weizman Institute of Science
}

Phys.Rev.Lett, in press, accepted 8/31/01 (astro-ph/0103275)

\begin{abstract}
Core collapse of massive stars resulting in a relativistic fireball jet which breaks through the stellar envelope is a widely discussed scenario for $\gamma$-ray burst production. For very extended or slow rotating stars, the jet may be unable to break through the envelope. Both penetrating and choked jets will produce, by photo-meson interactions of accelerated protons, a burst of $\gtrsim 5 \mathrm{TeV}$ neutrinos while propagating in the envelope. The predicted flux, from both penetrating and choked jets, should be easily detectable by planned $1 \mathrm{~km}^{3}$ neutrino telescopes.
\end{abstract}

PACS numbers: 96.40.Tv,98.70.Rz,98.70.Sa,14.60.Pq

The leading model for gamma-ray bursts (GRB) involves a relativistic fireball, where the $\gamma$-rays are produced by synchrotron or inverse Compton radiation from Fermi accelerated electrons in optically thin shocks. The ultimate energy source of the fireball is thought to be the gravitational energy release associated with temporary mass accretion onto a black hole, resulting either from a compact merger [1] or from the collapse of a massive star [2] (see [3] for a review). All the GRB so far localized with the Beppo SAX satellite [4] belong to the class of long bursts [5] with durations $\Delta t_{\gamma} \gtrsim 2 \mathrm{~s}$, and there is increasing evidence that these occur in star-forming galaxies [6]. For this class at least, comprising $\sim 2 / 3$ of all bursts, it is widely assumed that a massive, collapsing star ("collapsar") is the progenitor. The shocks producing the $\gamma$-rays must occur after the fireball has emerged from the stellar envelope. The preferred escape route is along the centrifugally lightened rotation axis, and the stellar pressure tends to collimate the fireball into a jet. The shocks should also lead to Fermi accelerated relativistic protons [7, 8], and to neutrinos with $\epsilon_{\nu} \gtrsim 10^{14} \mathrm{eV}$ via interactions with the $\sim \mathrm{MeV} \gamma$-rays [9, 10] (see [11] for a review).

In this Letter we show that while a jet is making its way through the star, its rate of advance is slowed down in a termination shock to a Lorentz factor much lower than it has at injection or after emergence; and additional internal shocks are expected in the jet interior, inward of the termination shock. The latter can accelerate protons to $\gtrsim 10^{5} \mathrm{GeV}$, which interact with X-ray photons in the jet cavity leading to electron and muon neutrinos (and anti neutrinos) with energies $\epsilon_{\nu} \gtrsim 5 \mathrm{TeV}$. These neutrinos appear as a precursor signal, lasting for time scales $\gtrsim$ tens of seconds prior to the observation of any $\gamma$-rays produced outside the star by a collapsar-induced GRB.

Furthermore, in a significant fraction of the massive stellar collapses, the jet may be unable to punch through the stellar envelope [12]. However, the $\mathrm{TeV}$ neutrino signal from such choked jets should be similar to that from "successful" jets which do break through and lead to observable GRB. This may provide a means of detecting and counting such choked-off, $\gamma$-ray dark collapses.

The TeV fluence from an individual collapse at cosmological distance $z \sim 1$ implies $10^{-1}-10$ upward moving muons per collapse/burst in a $1 \mathrm{~km}^{3}$ detector. The signal from an individual collapse with associated electromagnetic signals (and possibly from rare, energetic dark collapses), are well above the atmospheric neutrino background, and the total integrated flux from dark collapses should also be detectable. The AMANDA experiment 13,14 may soon be able to provide relevant limits on the total integrated rate of such dark collapses.

Jet propagation inside a massive progenitor envelope. Numerical simulations of collapsar models leading to black hole formation [12] have been performed for a range of stellar progenitors and initial conditions. The characteristic progenitor structure is that of an evolved massive star, with $\sim 2 M_{\odot}$ Fe core of radius $\sim 10^{9} \mathrm{~cm}$ and $\sim 8 M_{\odot}$ He core extending to $\sim 10^{11} \mathrm{~cm}$, in some cases surrounded by an $\mathrm{H}$ envelope extending to $\gtrsim 10^{13} \mathrm{~cm}$ (a red supergiant), while in others the envelope has been (largely) lost. While the details remain uncertain, preliminary calculations suggest that a relativistic jet can be launched along the progenitor rotation axis [12, 15], powered either by thermal $\nu \bar{\nu}$ annihilation or MHD stresses coupling to the black hole and to the debris disk falling back onto the hole. The jet life time is limited by the gas fall-back time onto the black hole, and should be comparable to GRB durations, $\lesssim 10^{2}$ s. (Similar pair or MHD outflows may also arise from collapses leading to highly magnetized pulsars [16, 17]).

When $\gamma$-rays are observed, the required isotropic equivalent luminosities are $L_{\text {iso. }} \gtrsim 10^{52} \mathrm{erg} / \mathrm{s}$ and the inferred jet Lorentz factors are $\Gamma_{j} \sim 10^{2}$. This is the final value outside the star, which may be representative of the intrinsic injection Lorentz factor. As it advances through the star, the jet drives a bow shock ahead of it. The jet is capped by a termination shock, and a re- 
verse shock moves back into the jet, at which the relativistic jet plasma is decelerated. Both the shocked jet plasma and the shocked stellar plasma advance with a jet head Lorentz factor $\Gamma_{h} \ll \Gamma_{j}$ [18]. In the rest frame of the shocked, decelerated plasma, the fast jet moves with a Lorentz factor $\approx \Gamma_{j} / 2 \Gamma_{h}$. During propagation in the $\mathrm{Fe}$ and He cores, the high density ahead of the jet implies deceleration of jet plasma to subrelativistic velocity. However, beyond the He core edge at $r \sim 10^{11} \mathrm{~cm}$, the density in an $\mathrm{H}$ envelope drops to $\rho \sim 10^{-7} \mathrm{~g} \mathrm{~cm}^{-3}$, and the jet can accelerate to relativistic velocity 18d (c.f. also [19]. Balancing the energy density behind the forward shock, $4 \Gamma_{h}^{2} \rho c^{2}$, with that behind the reverse shock, $4\left(\Gamma_{j} / 2 \Gamma_{h}\right)^{2} n_{j} m_{p} c^{2}$, where the jet proper proton number density $n_{j}=L_{\text {iso }} /\left(4 \pi r^{2} \Gamma_{j}^{2} m_{p} c^{3}\right)$, we find

$$
\Gamma_{h}=3 \frac{L_{52}^{1 / 4}}{r_{12}^{1 / 2} \rho_{-7}^{1 / 4}},
$$

where $L_{\text {iso }}=10^{52} L_{52} \mathrm{erg} / \mathrm{s}, r=10^{12} r_{12} \mathrm{~cm}$ is the shock radius, and $\rho=10^{-7} \rho_{-7} \mathrm{~g} \mathrm{~cm}^{-3}$.

The jet head propagates at mildly relativistic velocity $\Gamma_{h}$ up to a typical super-giant $\mathrm{H}$ envelope radius $r \sim 10^{13} \mathrm{~cm}$ (and if it makes it through entirely, in the even lower density beyond it would increase back to $\Gamma_{j} \sim 10^{2} \Gamma_{2}$ ). Envelopes larger than $R_{*} \gtrsim 10^{13} R_{13} \mathrm{~cm}$, or denser cores, e.g. due to slower rotation rate, could however lead to a stalling of the jet before it breaks through the star, if the jet crossing time is longer than the jet lifetime (as argued by, e.g. 12.18]).

The proper number density $n$ of protons and electrons in the shocked, decelerated jet plasma is related to the proper density $n_{j}$ in the fast moving jet through $n=4\left(\Gamma_{j} / 2 \Gamma_{h}\right) n_{j}$, and the energy density in the shocked plasma is $U=4\left(\Gamma_{j} / 2 \Gamma_{h}\right)^{2} n_{j} m_{p} c^{2}$. The hot, shocked jet plasma drives the bow shock wave that propagates forward (radially) and sideways (tangentially) into the envelope. For a jet of small opening angle $\theta<1 / \Gamma_{h}$ [18], the proper thickness of the shocked jet plasma shell is $\Delta \sim \theta r$. This can be seen by balancing the particle flux across the reverse shock, $\approx \pi(\theta r)^{2} c\left(\Gamma_{j} / 2 \Gamma_{h}\right) n_{j}$, with the tangential flux of particles leaving the cylinder of height $\Delta$ and radius $\theta r$ of shocked plasma, $\approx$ $2 \pi \theta r \Delta(c / \sqrt{3}) 4\left(\Gamma_{j} / 2 \Gamma_{h}\right) n_{j}$ (taking the tangential flow velocity to be comparable to the sound speed in the shocked plasma). Adopting $\Delta=0.2 \theta r$ and $\theta=10^{-1} \theta_{-1}$, the Thomson optical depth of the shocked plasma shell is

$$
\tau_{T} \approx 4 \times 10^{3} \frac{\theta_{-1} L_{52}}{r_{12} \Gamma_{2} \Gamma_{h}},
$$

where $\Gamma_{j}=10^{2} \Gamma_{2}$.

The reverse shock may start out being collisionless, heating the electrons and protons to $\sim 10^{2} \Gamma_{2} \mathrm{GeV}$. However, the electrons would lose all their energy on very short time scale, by synchrotron and inverse-Compton emission, converting a large fraction of the shocked plasma internal energy $U$ to radiation. Due to the high optical depth, the radiation will thermalize, with an approximate black body radiation temperature

$$
T_{r} \approx 4\left(\frac{L_{52}}{r_{12}^{2} \Gamma_{h}^{2}}\right)^{1 / 4} \mathrm{keV} .
$$

The corresponding proper photon number density is

$$
n_{\gamma} \approx 2 \times 10^{24}\left(\frac{L_{52}}{r_{12}^{2} \Gamma_{h}^{2}}\right)^{3 / 4} \mathrm{~cm}^{-3} .
$$

Thus, the reverse shock is likely to become radiation dominated, i.e. the dissipation of jet kinetic energy may be mediated by inverse-Compton scattering of the electrons, whose mean-free-path is $\sim 1 / n_{\gamma} \sigma_{T} \approx 1 \mathrm{~cm}$, where $\sigma_{T}$ is the Thomson cross section, and electrons lose all their momentum in a small number of scatterings (scattering of a thermal photon boosts its energy to $\sim 10\left(\Gamma_{2} / \Gamma_{h}\right)^{2} \mathrm{MeV}$ in the shocked plasma frame).

The mean-free-path of thermal photons propagating back into the jet, in the decelerated plasma frame, is

$$
l_{\gamma} \approx \Gamma_{h} /\left(2 \Gamma_{j} n_{j} \sigma_{T}\right)=5 \times 10^{6} \frac{\Gamma_{2} \Gamma_{h} r_{12}^{2}}{L_{52}} \mathrm{~cm} .
$$

Thus, the thermal photons do not penetrate much into the jet. If the reverse shock is indeed radiation dominated, the shock thickness would also be of order $l_{\gamma}$.

Photo-meson Interactions and Neutrinos. In addition to the bow and reverse shocks moving with $\Gamma_{h} \ll \Gamma_{j}$ in the star, internal shocks in the pre-deceleration jet moving with $\Gamma_{j}$ are expected to occur at radii

$$
r_{s} \approx \Gamma_{j}^{2} c \delta t=3 \times 10^{11} \Gamma_{2}^{2} \delta t_{-3} \mathrm{~cm},
$$

which are smaller than the termination and reverse shock radius. Here $\delta t$ is the variability timescale of the injected relativistic outflow, whose minimum value $\mathrm{s} \sim 10^{-3} \delta t_{-3}$ $\mathrm{s}$ 3,11]. While the details could depend on the ratio of MHD to pair and baryon energy, it is commonly assumed that internal shocks are mildly relativistic in the jet frame, and protons will be Fermi accelerated to a power law distribution which approximates $d n_{p} / d \epsilon_{p} \propto \epsilon_{p}^{-2}$ (see 11] for detailed discussion).

As they approach the reverse shock, high energy protons may produce pions in photo-meson interactions with thermal photons of energy $\epsilon_{\gamma} \sim T_{r} \sim 4 \mathrm{keV}$, provided their energy (measured in the decelerated plasma frame) satisfies $\epsilon_{p} \epsilon_{\gamma} \gtrsim 0.3 \mathrm{GeV}^{2}$, i.e. for $\epsilon_{p} \gtrsim 10^{5} \mathrm{GeV}$. The maximum energy to which protons may be accelerated in the internal shocks is limited by synchrotron losses. The acceleration time, in the jet frame, to observed energy $\epsilon_{p}^{\prime}=\Gamma_{h} \epsilon_{p}$, corresponding to energy $\approx \epsilon_{p}$ in the decelerated plasma frame, is approximately [7,11]

$$
t_{a} \sim \frac{R_{L}}{c}=\frac{\epsilon_{p}^{\prime}}{\Gamma_{j} e B}=10^{-6.5} \frac{\Gamma_{2}^{2} \Gamma_{h} \delta t_{-3}}{\left(L_{52} \xi_{B,-2}\right)^{1 / 2}} \epsilon_{p, 15} \mathrm{~s},
$$


while the (jet frame) time scale for synchrotron losses, which dominate at high energy, is

$$
t_{\mathrm{syn}}=\frac{6 \pi m_{p}^{4} c^{3} \Gamma_{j}}{\sigma_{T} m_{e}^{2} \epsilon_{p}^{\prime} B^{2}}=10^{2} \frac{\Gamma_{2}^{7} \delta t_{-3}^{2}}{\Gamma_{h} L_{52} \xi_{B,-2} \epsilon_{p, 15}} \mathrm{~s} .
$$

Here, $\epsilon_{p}=10^{15} \epsilon_{p, 15} \mathrm{eV}, R_{L}$ is the Larmor radius and $\xi_{B}=10^{-2} \xi_{B,-2}$ is the magnetic field equipartition fraction, $4 \pi r_{s}^{2} c \Gamma_{j}^{2} B^{2} / 8 \pi=\xi_{B} L_{\text {iso. }}$. Thus, protons may be accelerated to energies well above the pion threshold.

Protons at the threshold energy, for which the thermal photons are at the $\Delta$-resonance, lose $\approx 20 \%$ of their energy in each interaction. Inside the jet, the thermal photon density drops exponentially with the distance $x$ from the reverse shock, $n_{\gamma}(x) \propto \exp \left(-x / l_{\gamma}\right)$, and resonant protons will lose all their energy to pion production at a distance $x$ from the termination shock where the photon density satisfies

$$
n_{\gamma}^{\pi} \approx 5 /\left(l_{\gamma} \sigma_{p \gamma}\right)=2 \times 10^{21} \frac{L_{52}}{\Gamma_{2} \Gamma_{h} r_{12}^{2}} \mathrm{~cm}^{-3} .
$$

Here, $\sigma_{p \gamma}=5 \times 10^{-28} \mathrm{~cm}^{2}$ is the cross section at resonance. Typically, resonant protons and neutrons (which are produced with $\pi^{+}$'s in proton photo-pion interactions) will lose their energy at $x / l_{\gamma} \approx 10$, while higher energy nucleons, for which $\sigma_{p \gamma}$ is lower, will lose their energy to pions at shorter distance.

The pions produced in photo-meson interactions lose energy by inverse-Compton scattering on thermal photons. The lowest energy pions produced are characterized by Lorentz factors comparable to those of the resonant protons, $\approx 10^{5}\left(T_{r} / 4 \mathrm{keV}\right)^{-1}$ in the decelerated plasma frame. Inverse-Compton scattering of $\sim 4 \mathrm{keV}$ photons by pions of higher energy is in the Klein-Nishina regime. Thus, the inverseCompton energy loss time of high energy pions is $\tau_{I C} \approx$ $\left[3 n_{\gamma}^{\pi} \sigma_{T}\left(m_{e} / m_{\pi}\right)^{2} c / 8\right]^{-1}\left(\epsilon_{\pi} / 10^{13} \mathrm{eV}\right)\left(T_{r} / 4 \mathrm{keV}\right)$. The ratio of $\tau_{I C}$ to the pion life time is

$$
\frac{\tau_{I C}}{\tau_{\text {decay }}} \approx 5 \Gamma_{2} \Gamma_{h}^{1 / 2}\left(\frac{r_{12}^{2}}{L_{52}}\right)^{3 / 4} .
$$

We therefore expect $\approx 1 / 4$ of the energy lost by protons to pion production to be converted to muon neutrinos $(\approx 1 / 2$ of the energy is lost to neutral pion production, and $\approx 1 / 2$ of the charged pion energy is converted by decay into muon neutrinos).

If the termination shock is collisionless, rather than radiative, protons may be accelerated to energies exceeding the photo-meson threshold also in the termination shock. However, it is clear form Eq. (10) that inverse-Compton losses of the pions in the high density photon field prevent the production of very high energy neutrinos from protons accelerated in the termination shock.

The $\nu_{\mu}\left(\bar{\nu}_{\mu}, \nu_{e}, \bar{\nu}_{e}\right)$ from pion decays due to protons accelerated in internal shocks will have a typical energy
$5-10 \% \epsilon_{p}$. We therefore expect a flat power per decade neutrino spectrum [9], $\epsilon_{\nu}^{2} d n_{\nu} / d \epsilon_{\nu} \propto \epsilon_{\nu}^{0}$, at

$$
\epsilon_{\nu} \gtrsim 2\left(\frac{1+z}{2}\right)^{-1} \frac{\Gamma_{h}^{3 / 2} r_{12}^{1 / 2}}{L_{52}^{1 / 4}} \mathrm{TeV} .
$$

We note, that since photons propagating backward into the jet are emitted from a region of shocked, decelerated plasma with small optical depth, the spectrum of photons with which protons interact may differ from thermal. In particular, a non-thermal extension of the photon spectrum to energies $\gg T_{r}$ may lead to an extension of the neutrino spectrum to energies $<1 \mathrm{TeV}$. A detailed calculation of the photon spectrum is, however, beyond the scope of this Letter.

The jet isotropic-equivalent luminosity, $L_{\text {iso. }} \sim$ $10^{52} \mathrm{erg} / \mathrm{s}$, and energy, $E_{\text {iso. }} \sim 10^{53} \mathrm{erg}$, are inferred from $\gamma$-ray observations, and reflect the energy of electrons accelerated to high energy by the internal, mildlyrelativistic collisionless shocks. Protons are generally expected to be accelerated in such shocks with higher efficiency (see e.g. 22] for review). We therefore assume that the accelerated proton energy is at least comparable to that inferred from observations to be carried by accelerated electrons (see also [7, 9,23]). A large fraction of this accelerated proton energy will be converted, while the jet is inside the envelope, to pions. The envelope is transparent to $\mathrm{TeV}$ neutrinos, and the expected $\mathrm{TeV}$ neutrino fluence at Earth from a jet at a luminosity distance $D_{L} \sim 10^{28} \mathrm{~cm}$ (redshift $z \sim 1$ ) is

$$
F_{\nu_{\mu}\left(\bar{\nu}_{\mu}, \nu_{e}, \bar{\nu}_{e}\right.} \sim \frac{E / 8}{4 \pi D_{L}^{2}}=10^{-5} \frac{E_{53}}{D_{28}^{2}} \mathrm{erg} / \mathrm{cm}^{2},
$$

where $E_{\text {iso. }}=10^{53} E_{53}$ erg.

The probability that a muon neutrino will produce a high-energy upward moving muon in a terrestrial detector is approximately given by the ratio of the muon range to the neutrino mean free path [20], $P_{\nu \mu} \approx 1.3 \times$ $10^{-6} \epsilon_{\nu, \mathrm{TeV}}^{\beta}$, with $\beta=2$ for $\epsilon_{\nu, \mathrm{TeV}}<1$ and $\beta=1$ for $\epsilon_{\nu, \mathrm{TeV}}>1$. For a flat neutrino spectrum, this implies that the number flux $J_{\mu}$ of muon induced neutrinos can be related to the neutrino energy flux $F_{\nu}$ by $J_{\mu}=\left(P_{0} / \epsilon_{\nu, 0}\right) F_{\nu}$, with $P_{0} / \epsilon_{\nu, o}=1.3 \times 10^{-6} \mathrm{TeV}^{-1}$. Using Eq. (12), the average number of upward muon detections for an individual burst is

$$
N_{\mu} \approx \frac{P_{0}}{E_{0}} \frac{E / 4}{4 \pi D_{L}^{2}}=0.2 \frac{E_{53}}{D_{28}^{2}} \mathrm{~km}^{-2} .
$$

Implications. The typical angular resolution of planned neutrino telescopes at $\mathrm{TeV}$ energies (see [13] for a review) should be $\theta \sim 1$ degree. The atmospheric neutrino background flux is $\Phi_{\nu, b k g} \sim$ $10^{-7} \epsilon_{\nu, \mathrm{TeV}}^{-2.5} \mathrm{~cm}^{-2} \mathrm{~s}^{-1} \mathrm{ster}^{-1}$, implying a number of $>1 \mathrm{TeV}$ background events $N_{b k g} \sim 10^{-4}(\theta / \mathrm{deg})^{2} t_{2} \mathrm{~km}^{-2}$ per angular resolution element over a burst duration of $10^{2} t_{2} \mathrm{~s}$. 
Thus, the neutrino signals from individual collapses at $z \sim 1$ would stand out with high statistical significance above the background.

The rate of $\gamma$-ray-detectable GRB events, resulting from successful breakthroughs of jets beamed towards Earth, is $\sim 10^{3} /$ year 21. The predicted neutrino signal, $\sim 0.2 / \mathrm{km}^{2}$ muon events/burst at $\epsilon_{\nu_{\mu}} \gtrsim 5 \mathrm{TeV}$ [Eq. (13)], would precede by $t_{\text {cross }} \lesssim t_{j}=10^{2} t_{2}$ s the GRB trigger. Its detection would be a test of the collapsar hypothesis for long GRB, and the duration of the neutrino precursor would constrain the dimensions of, and jet advance speed in, the stellar envelope. In this case the absence (or a much reduced duration) of $\nu$-precursors in short GRB would be a test of the view that long and short GRB arise from qualitatively different progenitors.

The signals from both emerging and choked-off jets in equation (13) are calculated for "mean" events, i.e. for $E_{\text {iso }} \sim 10^{53} E_{53} \mathrm{erg}, \Gamma_{j} \sim 10^{2} \Gamma_{2}$ and $z \sim 1$. The total fireball jet energy and bulk Lorentz factors will, however, be distributed around these mean values, and fluctuations around the mean in these quantities and in the distance will lead to detections dominated by rare, energetic nearby events, as argued for GRB [23]. Thus, one could expect a significant number of neutrino bursts (few/year) with $N_{\mu} \gtrsim 10 / \mathrm{km}^{2}$ muon events/burst. These should be easily distinguishable above the atmospheric neutrino background at $z \sim 1$, whether coincident with observed GRB or not.

The number of collapsars with Earth-pointing choked jets, while model-dependent, may be in excess of the number of successful collapsar GRB (where jet punchthrough occurs and $\gamma$-rays are detected), $\gtrsim 10^{3} /$ year. If jet punch-through requires fast core rotation rate, e.g. from a massive star merger with a binary companion in a fraction of cases, choked collapsars could greatly exceed successful ones. The number of massive collapses may, e.g., be comparable to that of type II supernovae (SNe II), $\sim 3 \times 10^{-2} /$ year/galaxy. For a galaxy density $n_{G} \sim 10^{-2} / \mathrm{Mpc}^{3}$ within a Hubble radius $\sim 3 \mathrm{Gpc}$ $(z \sim 1)$ the total rate of SNe II is $\mathcal{R}_{S N} \sim 1 \mathrm{~s}^{-1}$. If all SNe II lead to jets beamed into a solid angle $\Omega_{j} \sim 10^{-2} 4 \pi$ (e.g. 24, 16, 25), the effective rate beamed towards Earth would be $\sim 10^{5}$ /year. From equation (12), the $\nu \bar{\nu}$ energy flux per logarithmic energy interval from all directions would be $\epsilon_{\nu}^{2} \Phi_{\nu} \sim 10^{-7} \mathrm{GeV} / \mathrm{cm}^{2} \mathrm{ssr}$, where we divided by $\ln \left(\epsilon_{\nu, \max } / \epsilon_{\nu, \min }\right) \sim 10$. This signal is not far below the experimental upper bound on the diffuse neutrino flux recently established by the AMANDA experiment, $\epsilon_{\nu}^{2} \Phi_{\nu} \leq 10^{-6} \mathrm{GeV} / \mathrm{cm}^{2}$ s sr 14. An increase in neutrino telescope exposure will therefore allow in the near future to put relevant constraints on the frequency of $\gamma$-ray dark $\nu$-bursts from choked jets, and hence on the frequency of core collapses at the poorly known high end of the stellar mass distribution. (Unfavorably aligned emerging jets would also appear $\gamma$-ray dark, but would be neutrinodark as well).
The TeV neutrino signals preceding the $\gamma$-rays in GRB, as well as those from choked, $\gamma$-ray dark jets, would differ significantly from other neutrino signals expected during and after the $\gamma$-ray phase of GRB. In the latter, one expects $\gtrsim 100 \mathrm{TeV}$ neutrinos [9] from internal shocks well beyond the stellar envelope accelerating protons that interact with $\mathrm{MeV}$ photons, or $\gtrsim 10^{17} \mathrm{eV}$ neutrinos 26. from external shock protons interacting with UV photons even further out, and one also expects $2-5 \mathrm{GeV}$ neutrinos 2729 from inelastic nuclear collisions when neutrons decouple from protons. For the flat $\epsilon_{\nu}^{2} \Phi_{\nu}$ neutrino spectra considered here, the number of precursor $\mathrm{TeV}$ neutrino events per burst is also larger by at least one order of magnitude, relative to those at $\gtrsim 100 \mathrm{TeV}$ and at $\mathrm{GeV}$ expected during and after the $\gamma$-bright phase of GRB. Such GRB precursor or $\gamma$-ray dark TeV neutrino burst signals may therefore be the likeliest targets for early detection with planned $\mathrm{km}^{3}$ experiments 13 .

If, as suspected, the first generations of stars formed in the Universe are much more massive than those forming now, then at redshifts $z \gtrsim 5$ jet break-through collapses may be rare, and $\gamma$-ray dark choked jet collapses leading to TeV neutrinos may be much more common than today. Without an emerging jet, the $\mathrm{X}$-ray, optical, IR and radio afterglows typical of GRB would also be absent. Such neutrino bursts, which at $z \sim 5$ produce a $\gtrsim 1 \mathrm{TeV}$ fluence smaller by only a factor of $\approx 10$ compared to $z \sim$ 1 bursts, would therefore be a prime tool for investigating both successful and failed GRB, as well as massive star formation at such high redshifts.

Acknowledgements: Partial support was received from BSF 9800343, Universities Planning \& Budget Committee, Israel, NSF AST0098416 and NASA NAG5-9192.

[1] Paczyński , B., 1986, ApJ, 308, L43; Goodman, J., 1986, ApJ, 1986, 308, L47.

[2] Woosley, S., 1993, Ap.J., 405, 273; Paczyński , B., 1998, ApJ, 494, L45.

[3] Mészáros , P, 2001, Science, 291, 79

[4] Costa, E, 1999, in Gamma Ray Bursts, eds. R. Kippen at al., AIP Conf/ Proc. 526, p. 365

[5] Kouveliotou, C., et al., 1993, Ap.J., 413, L101

[6] Bloom, J, Kulkarni, R \& Djorgovski, G, 2000, ApJ subm (astro-ph/0010176

[7] Waxman, E, 1995, PRL, 75, 386

[8] Vietri, M, 1995, ApJ, 453, 883

[9] Waxman, E \& Bahcall, JN 1997, Phys Rev Lett. 78, 2292; Waxman, E \& Bahcall, JN, 1999, Phys.Rev. D 59, 023002

[10] Rachen, J \& Mészáros , P, 1998, Phys Rev D, 58, 123005

[11] Waxman, E 2001, Nuc. Phys. B (Proc. Supp.) 91, 494

[12] MacFadyen, A, Woosley, S \& Heger, A, 2001, ApJ (astro$\mathrm{ph} / 9910034$ ) 
[13] Halzen, F, 2001, in Intl Symp on High Energy Gamma Ray Astronomy, Heidelberg, June 2000 astroph/0103195)

[14] Andres, E. et al., 2001, Nature, 410, 441

[15] Aloy, M, et al, 2000, ApJ 531, L119

[16] Cen, R, 1998, ApJ 507, L131

[17] Wheeler, J.C., Yi, I; Hoefflich, P. \& Wang, L., 2000, ApJ 537,810

[18] Mészáros , P \& Rees, MJ, 2001, ApJ, 556, L37

[19] Tan, J, Matzner, CD, McKee, CF, 2001, ApJ 551, 946

[20] Gaisser, T, Halzen, F \& Stanev, T, 1995, Phys.Rep. 258, 173

[21] Fishman, G.J. \& Meegan, C.A. 1995, ARAA, 33, 415; van Paradijs, J, Kouveliotou, C \& Wijers, R, 2001, ARAA, in press

[22] Blandford, R., \& Eichler, D., Phys. Rep. 154, 1 (1987).

[23] Alvarez-Muniz J, Halzen F, Hooper D, 2000, PRD 62:093015

[24] Frail, D, et al, Nature, subm (astro-ph/0102282)

[25] Wang, L, Wheeler, JC, 1998, ApJ 504, L87

[26] Waxman, E \& Bahcall, JN 2000, ApJ, 541, 707

[27] Bahcall, JN \& Mészáros , P, 2000, PRL, 85, 1362

[28] Mészáros , P. \& Rees, M.J. 2000, ApJ(Lett.), 541, L5

[29] Derishev, EV, Kocharovsky, VV \& Kocharovsky, Vl.V 1999, ApJ 521, 640 